\title{
Experimental Realization of Self-Guiding Unidirectional Electromagnetic Edge States
}

\author{
Yin Poo, ${ }^{1}$ Rui-xin Wu, ${ }^{1, *}$ Zhifang Lin, ${ }^{2}$ Yan Yang, ${ }^{1}$ and C. T. Chan ${ }^{3}$ \\ ${ }^{1}$ School of Electronic Science and Engineering, Nanjing University, Nanjing 210093, China \\ ${ }^{2}$ Key Laboratory of Micro and Nano Photonic Structures (Ministry of Education) and Department of Physics, \\ Fudan University, Shanghai 200433, China \\ ${ }^{3}$ Department of Physics, Hong Kong University of Science and Technology, Clear Water Bay, Hong Kong, China \\ (Received 24 September 2010; revised manuscript received 22 January 2011; published 2 March 2011)
}

\begin{abstract}
We present an experimental demonstration of self-guiding electromagnetic edge states existing along the zigzag edge of a honeycomb magnetic photonic crystal. These edge states are shown to possess unidirectional propagation characteristics that are robust against various types of defects and obstacles. In particular, they allow for the unidirectional transport of electromagnetic energy without requiring an ancillary cladding layer.
\end{abstract}

DOI: 10.1103/PhysRevLett.106.093903

PACS numbers: 42.70.Qs, 41.20.Jb, 78.20.Ls

Chiral edge states (CESs) in the quantum Hall effect (QHE) have attracted much attention [1-5]. The CESs carry current in one direction and the transport is robust against scattering from disorder [6,7]. Such one-way states are usually realized by applying a strong magnetic field perpendicular to two-dimensional (2D) electron systems to break time-reversal symmetry (TRS). Inspired by the analogy between electronic and photonic systems, Raghu and Haldane [8,9] predicted the existence of electromagnetic (EM) one-way edge modes in 2D photonic crystals (PCs). When a static bias magnetic field (BMF) is applied, the breaking of TRS opens a photonic band gap at the Dirac points, giving rise to states that are the photonic analogues of electronic CESs [8-11]. Similar to the electronic systems, the photonic edge states are confined to the area near the edge of the 2D PC, exhibiting one-way propagation characteristics.

However, there is an important difference between electronic CESs and their photonic analogues. Electronic states can have negative energies, and can thus be naturally bounded; while photonic states usually suffer from radiation leakage, unless the system configuration specifically forbids the coupling with vacuum modes [10]. As a result, previous realizations of one-way transport require an ancillary cladding layer made of either a perfect metal or PC [12-14] to confine the CESs to the edge. As pointed out by Wang et al. [12], without the ancillary cladding, the photonic CESs at the edges of the PC will disappear, instead of running along the "one way road of light" [15]. In this sense, the one-way propagating modes that were observed $[12,13]$ are one-way waveguide modes and the unidirectionality in propagation is facilitated by a TRS broken system that constitutes one of the waveguide boundaries to suppress the backward propagation.

Motivated by the experimental observation of an unusual half-integer QHE in graphene [1] and the prediction of edge states in honeycomb plasmonic lattices [16], we explored experimentally the possibility of the existence of one-way photonic edge states that are guided wave modes on the edge and are evanescent in free space [17]. These edge states have been demonstrated to exhibit unidirectionality in propagation along the edge of PCs and show robustness against scattering or disorder. In particular, these EM edge states enable the subwavelength transport of the EM energy without having to resort to the confinement of radiation by an ancillary cladding layer $[12,13]$. Their self-guiding features may open up new avenues for applications in the emerging field of unidirectional light propagation $[8-15,18,19]$.

Our system is a magnetized 2D honeycomb magnetic photonic crystal (MPC) operating for the transverse magnetic modes, with the $E$-field polarized along the rod axis, defined as the $z$ axis. Several rows of ferrite rods are arranged in a honeycomb lattice as shown in Fig. 1(a). The lattice structure is isomorphic to electronic graphene [1]. As our actual sample has a finite width, we follow the graphene literature to call our sample a "ribbon." Each rod has a radius of $2 \mathrm{~mm}$ and height of $10 \mathrm{~mm}$. The lattice constant $a$ is $10 \mathrm{~mm}$. Commercially available yttrium iron garnet (YIG) ferrite is used. The ferrite has a saturation magnetization $4 \pi M_{s}=1884 \mathrm{G}$ and a resonance linewidth $\Delta H=50$ Oe. Its permittivity is about 15.26-0.003i, which is essentially constant at the microwave frequencies.

We first present the band structure calculations of the MPC, calculated using a multiple-scattering method [20]. In the magnetized state as shown in Fig. 1(a), the fully magnetized ferrite has a permeability tensor given by [21]:

$$
\overline{\bar{\mu}}=\mu_{0}\left(\begin{array}{ccc}
\mu & i \kappa & 0 \\
-i \kappa & \mu & 0 \\
0 & 0 & 1
\end{array}\right),
$$

where $\mu=1+\frac{\omega_{m}\left(\omega_{0}+i \alpha \omega\right)}{\left(\omega_{0}+i \alpha \omega\right)^{2}-\omega^{2}}$ and $\kappa=\frac{\omega_{m} \omega}{\left(\omega_{0}+i \alpha \omega\right)^{2}-\omega^{2}}$. Here, $\omega_{0}=\gamma H_{0 i}$ is the resonance frequency, $\omega_{m}=4 \pi \gamma M_{s}$ is the characteristic frequency, and $\gamma$ is the gyromagnetic ratio. $H_{0 i}$ is the effective magnetic field in ferrite rods, 

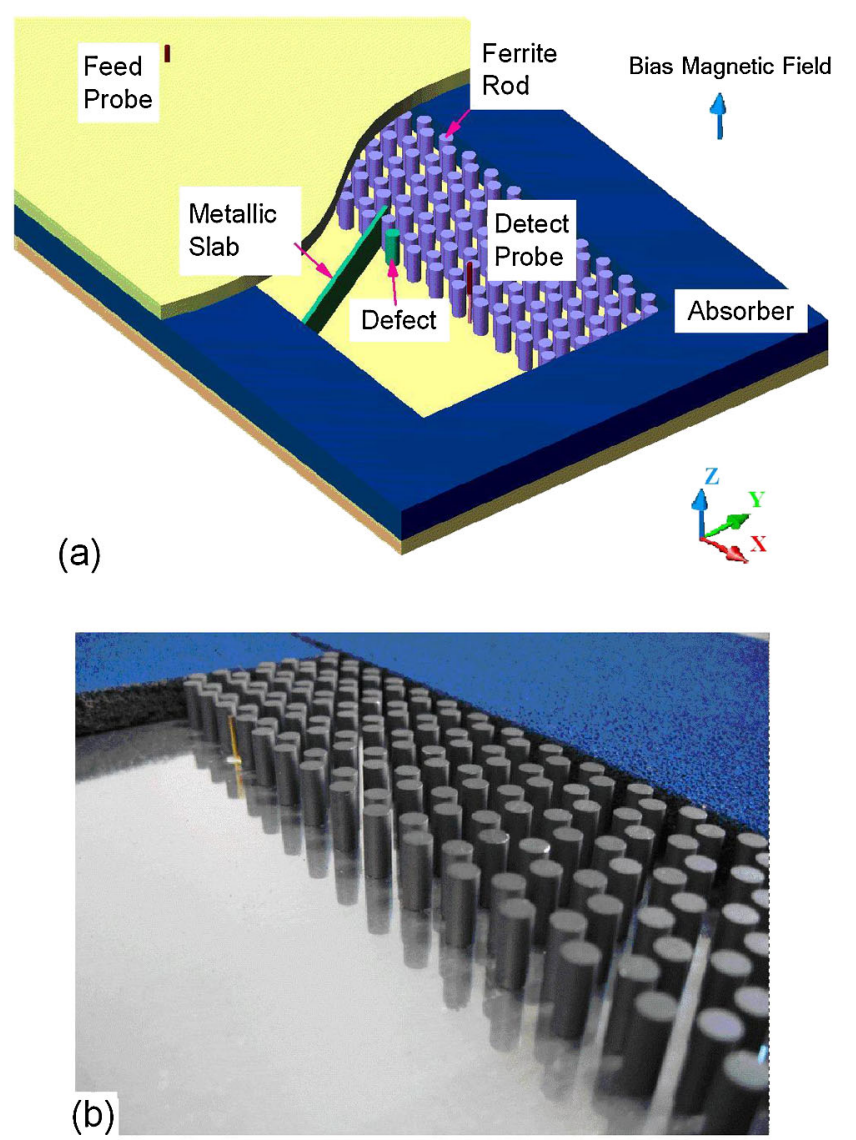

FIG. 1 (color online). (a) A schematic picture of the ferrite array sample inside the parallel plate waveguide. The waveguide is sandwiched between two metallic plates and surrounded by the absorber. To test the robustness of the wave propagation, defects in the form of a metal slab or a rod are added to the zigzag edge. (b) A closer look at the fabricated sample with the top plate removed.

which is the sum of the BMF and the demagnetization field. The off-diagonal element in the permeability tensor, which is induced by the nonzero BMF, breaks the TRS and the degree of breakage is characterized by the ratio of $\kappa$ to $\mu$

Figure 2(a) shows the band structure projected along the zigzag axis of the MPC at $H_{0 i}=800$ Oe. A gap between 7.75 and $8.42 \mathrm{GHz}$ is identified. When the zigzag edge is exposed to air, edge states appear inside the band gap as shown in Fig. 2(b), the band structure of a ferrite array ribbon. The red (dark gray) curves correspond to edge states localized on one side of the sample while the green (light gray) curves correspond to edge states localized on the other side. The regions marked with numbers 1 and 2 denote the frequency range where the edge states exist outside the light cone, while region 2 also denotes the frequency range where the edge modes appear with $k a<$ $2 \pi / 3$. The edge states that exist outside the light cone allow for the self-guiding feature along the edge. In other words, the states are naturally evanescent in air and do not

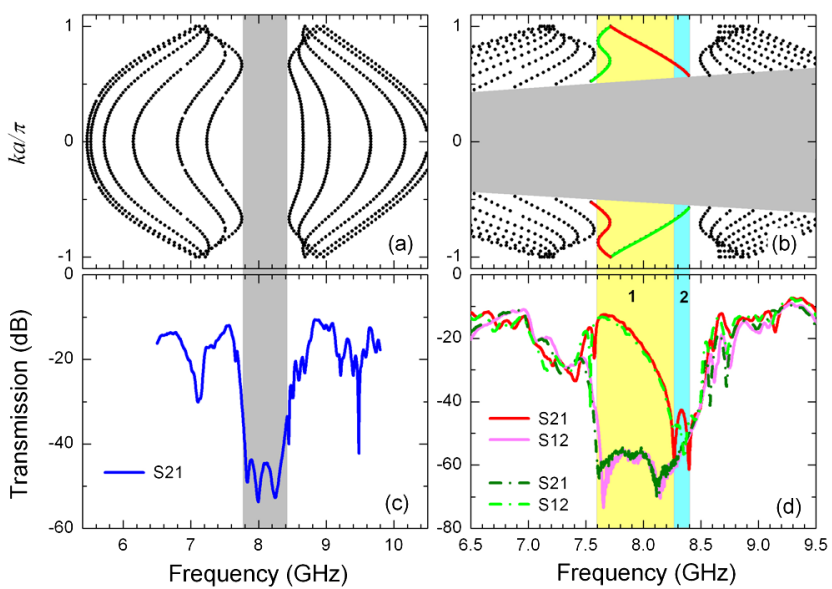

FIG. 2 (color online). The band structure of (a) the bulk MPC and (b) the MPC ribbon projected along the zigzag axis $(\Gamma-\mathrm{K})$. The light cone is shaded and the edge states are shown in red (dark gray) and green (light gray). (c) The unprocessed transmission data for the bulk MPC (data presented as is, without renormalization); (d) the unprocessed transmission data measured at the zigzag edge exposed to air. The transmissions denoted by the solid and dashed lines in panel (d) are obtained with opposite direction of the BMF.

require an auxiliary boundary (e.g., a gapped material or a metal wall) to confine the radiation.

To characterize the properties of the self-guiding edge states, we refer again to the experimental setup in Fig. 1(a). The sample is surrounded by absorbers with only one zigzag edge exposed to air. This edge is predicted to support one-way propagation. Figure 1(b) shows a picture of the actual sample which has five chains, each consisting of 39 ferrite rods. The ribbon sample is placed to occupy part of the chamber surrounded by the absorber. Simulations showed that a ribbon containing five chains is good enough to demonstrate the photonic CESs. The feed and detect probes are $130 \mathrm{~mm}$ apart and are located at the zigzag edge, as shown in Fig. 1(a). The sample is sandwiched between two parallel metallic plates with a gap of $10 \mathrm{~mm}$, which supports TEM mode waves of up to $15 \mathrm{GHz}$. The EM wave is fed into the waveguide by the feed probe and is detected by the detect probe along the $x$ axis. In the waveguide, the $E$ field is polarized along the $z$ axis. The transmission measurements were made with the Agilent E8363A vector network analyzer. The uniform BMF along the $z$ axis was provided by a big Helmholtz coil with an inner diameter of $260 \mathrm{~mm}$, which is large enough to guarantee a spatial uniform BMF.

Experimentally, we first characterized the band gap of a bulk MPC by using an MPC sample that filled the whole chamber. The two probes $130 \mathrm{~mm}$ apart were inserted into the interior of the sample and set far away from the edges of the MPC to avoid the influence of the boundary. Figure 2(c) shows the results measured at $H_{0 i}=800$ Oe. The band gap has a $40 \mathrm{~dB}$ extinction for the transmission, 
and its frequency range agrees well with the calculated band structure shown in Fig. 2(a).

Next, we identified the self-guiding edge states and characterized their one-way propagation property. For edge modes, energy transport is allowed only in one direction due to the breaking of TRS. Therefore, the transmission coefficients S21 and S12 (representing forward and backward propagation, respectively) should show strong contrast. The solid lines in Fig. 2(d) denote the unprocessed transmission (data presented as is, without renormalization) at $H_{0 i}=800$ Oe measured using the configuration shown in Fig. 1(a), with the BMF along the positive $z$ direction. Between 7.59 to $8.25 \mathrm{GHz}$, the transmission coefficient $\mathrm{S} 21$ is much greater than $\mathrm{S} 12$, showing that the wave propagates along the positive $x$ direction and is evanescent along the negative $x$ direction. The biggest difference between S21 and S12 is approximately $60 \mathrm{~dB}$ in the vicinity of $7.65 \mathrm{GHz}$. The frequency range of one-way propagation is in good agreement with that of the zigzag edge mode in the band structure when $k a>2 \pi / 3$ [see Fig. 2(b)], consistent with the fact that edge modes are highly localized on the edge if $k a>2 \pi / 3$. However, if $k a<2 \pi / 3$, the edge modes are less localized [16].

In addition, we switched the direction of the external field to the negative $z$ direction and the results are shown in Fig. 2(d) in dashed lines. The measured transmissions are quite similar to the previous ones, but the curves of S21 and S12 have swapped so that the wave is now propagating along the negative $x$ direction. Our results experimentally prove the existence of the self-guiding edge states along the zigzag edge of a MPC [18]. We also note that edge states exist at higher frequencies [region 2 in Fig. 2(b)] where $k a<2 \pi / 3$. The measurement showed that the EM wave propagates unidirectionally in this region, but the difference between the forward and backward propagation is smaller than that of the CESs with $k a>$ $2 \pi / 3$. Our calculations indicate that the edge states in this regime penetrate deeper into the bulk of the MPC, resulting in the decreased transmission measured by the probe located at the edge. Similar phenomena have been observed in the plasmonic case [16].

The one-way propagation is expected to be robust against scattering by disorder $[2,8,9,12-14,18,19]$ for the lack of a backscattering channel. This property holds for the self-guiding edge mode demonstrated here as well. The characteristics of these edge states are presented in our online materials [22], where we also highlight the difference between the self-guiding zigzag edge states and the leaky edge states previously reported [12].

To demonstrate experimentally the robustness of oneway transport in the self-guiding edge modes, we measured the transmission of the sample under the influence of obstacles that are placed either close to or directly on the zigzag edge. Figure 3 shows the results of placing an obstacle near the edge. The obstacles used include a piece of absorber [Fig. 3(a)] and a metal block [Fig. 3(b)] of size $2 a$, as schematically shown in the inset of Fig. 3. Compared

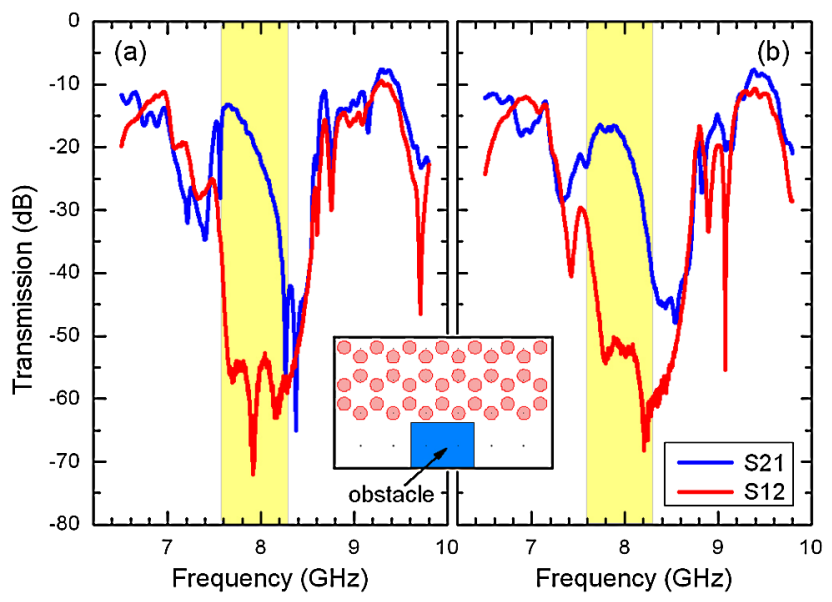

FIG. 3 (color online). The unprocessed transmission data measured when (a) an absorber, (b) a metal block is placed near the edge of the MPC, as illustrated in the inset.

with Fig. 2(d), the transmission characteristics remain almost the same, although there is a small change in the magnitude. The difference between S21 and S12 is still large, about $53 \mathrm{~dB}$ in the vicinity of $7.91 \mathrm{GHz}$. Although the scattering properties of the two obstacles are quite different, the one-way propagation characteristic remains evident, showing that the unidirectional transport is not significantly affected by scatterers placed near the edge of the sample because the field is confined within the subwavelength lateral scales on the edge.

Figure 4 shows that the power transmission in the selfguiding edge states is hardly affected by scattering from defects placed directly on the edge [12]. Figure 4(a) shows the measured transmission when a ferrite rod at the edge is replaced by a metallic rod as illustrated in Fig. 4(b) and Fig. 4(c) shows the result when a ferrite rod is removed as illustrated in Fig. 4(d). The forward transmission is still much larger than the backward transmission in the presence of different defects. The simulation results show that instead of being backscattered, most of the EM energy will circumvent the defect and keep propagating as shown in Figs. 4(b) and 4(d). For the metal rod, the power flow swirls around the ferrite rods and there is little radiation leakage near the metallic rod. The forward transmission is slightly $(1 \mathrm{~dB})$ larger than that when one ferrite rod is removed from the edge. The results show that the one-way propagation is robust against defect scattering, even without any external cladding.

We then inserted a metallic slab into the zigzag edge [see Fig. 4(f)] to block the guided wave and Fig. 4(e) gives the measured transmissions. The strong forward transportation is still preserved. The simulation results in Fig. 4(f) show that the power flows around the inserted slab. Figures $4(\mathrm{~g})$ and $4(\mathrm{~h})$ give the measured transmission when a metallic slab is inserted deeper into the MPC to block two and three zigzag chains, respectively. The S21 and S12 still exhibit a sharp contrast, preserving the unidirectional propagation. As the rerouting extends the propagation path of the guided 


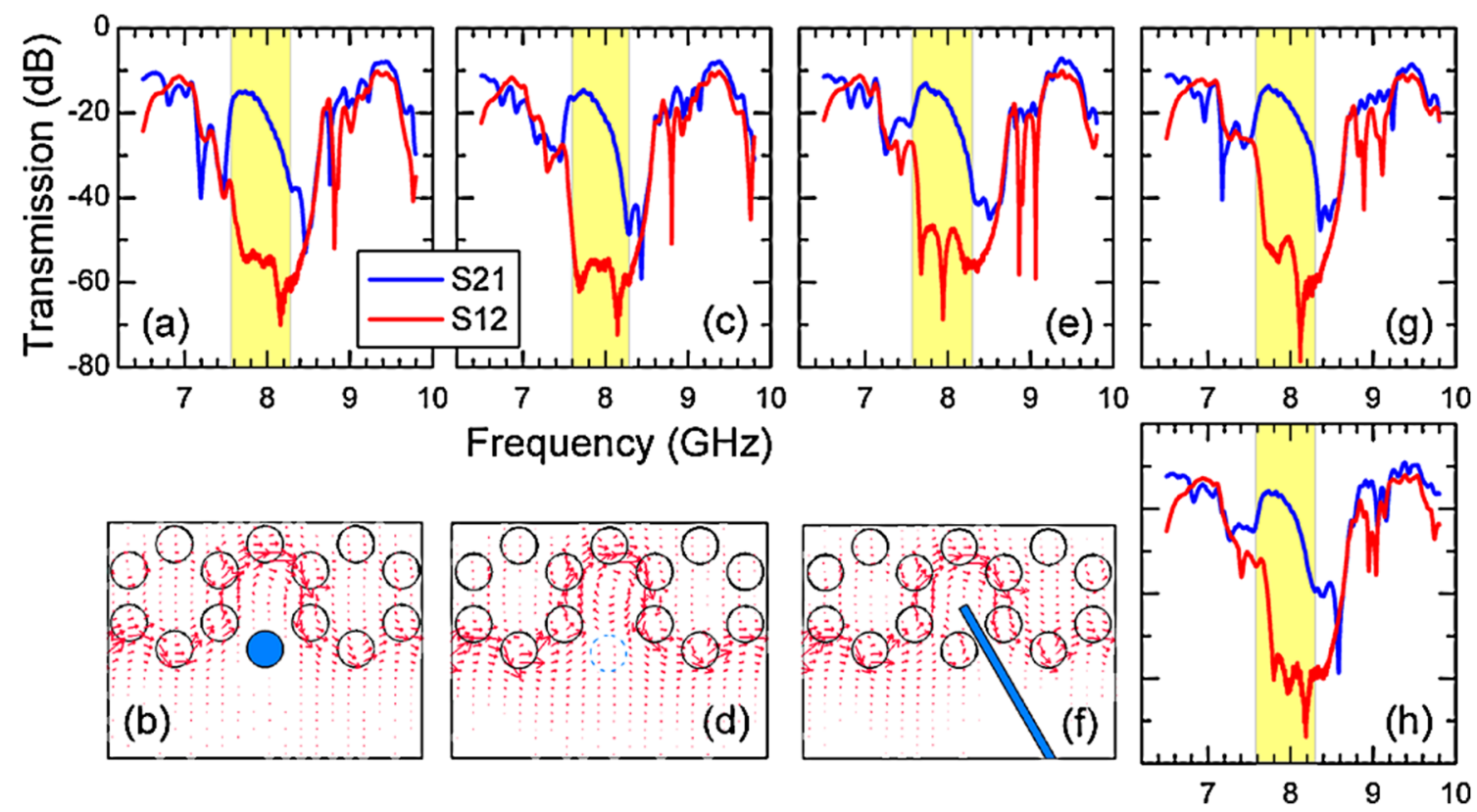

FIG. 4 (color online). The unprocessed transmission data measured with different types of disruptions on the zigzag edge. (a) Measured transmission and (b) simulated power flow when a ferrite rod on the edge is replaced by a metallic rod of the same size; (c) Measured transmission and (d) simulated power flow when a ferrite rod on the zigzag edge is removed; (e) Measured transmission and (f) simulated power flow when the first zigzag chain is blocked by a metallic slab; Panels (g) and (h) illustrate, respectively, measured transmission when two and three zigzag chains are blocked.

wave along the edge, the amplitude of the forward wave is slightly reduced because of the damping effect of ferrite and the increased radiation leakage. The figures illustrate that the backward wave is still evanescent, and damps rapidly as the distance increases. Although the details vary with the type of defect, the EM wave persists in moving forward by circumventing the defect as it has no backward channel and the leakage into air is found to be small for the defects we have considered. Therefore, the transmission is robust against defects placed directly on the edge, corroborating with the simulation results [22].

In conclusion, we have experimentally demonstrated the existence of self-guiding EM edge states along the zigzag edge of a honeycomb magnetic photonic crystal. The oneway propagation in a bias magnetic field and the robustness against imperfections on or near the edge are also demonstrated. Such self-guiding edge states offer the possibility of guiding EM wave unidirectionally without using any ancillary confinement claddings.

This work is supported by the NSFC (60771013, 61071007, 10774028), the China 973 program (2011CB922004), MOE of China (B06011), and SMCST (08dj1400302). Work conducted in Hong Kong is supported by the RGC of Hong Kong (600308).

*rxwu@nju.edu.cn

[1] Y. Zhang et al., Nature (London) 438, 201 (2005).

[2] R. E. Prange and S. M. Girvin, The Quantum Hall Effect (Springer-Verlag, Berlin, 1987).
[3] D. C. Tsui, H. L. Stormer, and A. C. Gossard, Phys. Rev. Lett. 48, 1559 (1982).

[4] K. v. Klitzing, G. Dorda, and M. Pepper, Phys. Rev. Lett. 45, 494 (1980).

[5] K.S. Novoselov et al., Nature (London) 438, 197 (2005).

[6] D. J. Thouless et al., Phys. Rev. Lett. 49, 405 (1982).

[7] Y. Hatsugai, Phys. Rev. Lett. 71, 3697 (1993).

[8] S. Raghu and F. D. M. Haldane, Phys. Rev. A 78, 033834 (2008).

[9] F. D. M. Haldane and S. Raghu, Phys. Rev. Lett. 100, 013904 (2008).

[10] T. Ochiai and M. Onoda, Phys. Rev. B 80, 155103 (2009).

[11] S. Bittner et al., Phys. Rev. B 82, 014301 (2010).

[12] Z. Wang et al., Nature (London) 461, 772 (2009).

[13] J.X. Fu, R. J. Liu, and Z. Y. Li, Appl. Phys. Lett. 97, 041112 (2010).

[14] Z. Wang et al., Phys. Rev. Lett. 100, 013905 (2008).

[15] E. Yablonovitch, Nature (London) 461, 744 (2009).

[16] D. Han et al., Phys. Rev. Lett. 102, 123904 (2009).

[17] The EM modes that are guided in a specific direction while being evanescent in free space are sometimes called "dark modes"; see, for example, M.Z. Liu et al., Phys. Rev. Lett. 102, 107401 (2009).

[18] X. Y. Ao, Z.F. Lin, and C. T. Chan, Phys. Rev. B 80, 033105 (2009).

[19] S. Y. Liu et al., Appl. Phys. Lett. 97, 201113 (2010).

[20] S. Y. Liu et al., Phys. Rev. Lett. 101, 157407 (2008).

[21] D. M. Pozar, Microwave Engineering (Wiley, New York, 2005), 3rd ed.

[22] See supplemental material at http://link.aps.org/ supplemental/10.1103/PhysRevLett.106.093903 for the characteristics of the self-guiding zigzag edge states. 